\title{
On solvability of the Neumann boundary value problem for a non-homogeneous polyharmonic equation in a ball
}

\author{
Batirkhan K Turmetov ${ }^{1 *}$ and Ravshan R Ashurov²
}

${ }^{*}$ Correspondence:
turmetovbh@mail.ru
'Department of Mathematics,
Akhmet Yasawi International
Kazakh-Turkish University,
B. Sattarkhanov street, 29, Turkistan,
161200, Kazakhstan
Full list of author information is
available at the end of the article

available at the end of the article

\begin{abstract}
In this work the Neumann boundary value problem for a non-homogeneous polyharmonic equation is studied in a unit ball. Necessary and sufficient conditions for solvability of this problem are found. To do this we first reduce the Neumann problem to the Dirichlet problem for a different non-homogeneous polyharmonic equation and then use the Green function of the Dirichlet problem.

MSC: Primary 35J40; secondary 35J30; 35A01
\end{abstract}

Keywords: non-homogeneous polyharmonic equation; the Neumann problem; the necessary and sufficient conditions for solvability

\section{Introduction}

Let $\Omega=\left\{x \in R^{n}:|x|<1\right\}$ be a unit ball, $\partial \Omega=\left\{x \in R^{n}:|x|=1\right\}$ be a unit sphere and $m$ be a positive integer.

Consider on the domain $\Omega$ the following Neumann boundary value problem:

$$
\begin{aligned}
& (-\Delta)^{m} u(x)=g(x), \quad x \in \Omega, \\
& \frac{\partial^{k} u}{\partial \nu^{k}}(x)=\varphi_{k}(x), \quad k=1,2, \ldots, m, x \in \partial \Omega,
\end{aligned}
$$

where $v$ is the unit outer normal vector to sphere $\partial \Omega, g(x)$ and $\varphi_{k}(x)$ are given functions; we always suppose that these functions are sufficiently smooth, and from here on we do not pay any attention to their smoothness.

A function $u(x) \in C^{2 m}(\Omega) \cap C^{m+1}(\bar{\Omega})$ is called a solution of problem (1), (2) if it satisfies (1), (2) in a classical sense.

It is well known (see, for example, [1]) that even in case $m=1$ the considered problem (1), (2) does not have solutions for arbitrary (even, as we supposed, smooth) functions $g(x)$ and $\varphi_{k}(x)$; in the case of the Poisson equation, the necessary and sufficient solvability condition for the Neumann problem is

$$
\int_{\Omega} g(x) d x=\int_{\partial \Omega} \varphi_{1}(x) d S_{x} .
$$

C 2013 Turmetov and Ashurov; licensee Springer. This is an Open Access article distributed under the terms of the Creative Commons Attribution License (http://creativecommons.org/licenses/by/2.0), which permits unrestricted use, distribution, and reproduction in any medium, provided the original work is properly cited. 
In the paper [2] by Kanguzhin and Koshanov, in particular, it is shown that in case $m=2$ the necessary and sufficient condition for solvability of problem (1), (2) has the form

$$
\begin{aligned}
& \int_{\partial \Omega}\left\{\varphi_{1}(x)-\varphi_{2}(x)+\int_{\Omega} d_{2, n}(4-n)\right. \\
& \left.\quad \times\left[(2-n)|x-y|^{-n}(1-(x, y))^{2}+|x-y|^{2-n}(x, y)\right] g(y) d y\right\} d S_{x}=0,
\end{aligned}
$$

where $(x, y)$ is the scalar product in $R^{n}$ and $d_{2, n}=\frac{\Gamma\left(\frac{n}{2}-2\right)}{16 \pi \frac{n}{2}}$.

The authors of the paper [3] presented this condition in a different form, which could be easily verified,

$$
\int_{\Omega} \frac{1-|x|^{2}}{2} g(x) d x=\int_{\partial \Omega}\left[\varphi_{2}(x)-\varphi_{1}(x)\right] d S_{x}
$$

In the above paper [2] the authors found a solvability condition for Neumann problem (1), (2) for arbitrary $m$ as well (see [2], Theorem 4.2). This condition follows from equality to zero of the determinant of an $m \times m$ matrix, one column of which consists of integrals $\int_{\partial \Omega}\left[\varphi_{k}(x)-\partial^{k} / \partial \nu^{k}\left(\varepsilon_{m, n} * g(x)\right)\right] d S_{x}, \varepsilon_{m, n}=d_{m, n}|x|^{2 m-n}$ and $d_{m, n}$ is a constant. Note that the equation which one has as a result is very difficult to verify.

The main goal of the present paper is to find a solvability condition for problem (1), (2) in a more simple form. It should be noted that in our study of problem (1), (2) the Green function of the Dirichlet problem for equation (1) is essentially used. In the paper [4] a similar method was used in the solution of the boundary value problem for the Poisson equation with the boundary operator of fractional-order.

The paper is organized as follows. In the next section we study the properties of some integro-differential operators, which we then use throughout the paper. In Section 3 we investigate the Dirichlet problem for a polyharmonic equation, making use of the explicit form of the Green function found in [5-7]. Then, in the following section, reducing Neumann problem (1), (2) to the considered Dirichlet problem, we give the necessary and sufficient solvability condition for problem (1), (2) with homogeneous boundary conditions. In the same way we consider in Section 5 the Neumann boundary value problem for the homogeneous equation with non-homogeneous boundary conditions. Finally, in Section 6 we study problem (1), (2) in the general case. To present the necessary and sufficient conditions for solvability, here we apply the Almansi formula for constructing solutions to the Dirichlet problem.

\section{Properties of some integro-differential operators}

Let $u(x)$ be a sufficiently smooth function in $\Omega$. Consider the following operators:

$$
\Gamma_{c}[u](x)=\left(r \frac{\partial}{\partial r}+c\right) u(x), \quad \Gamma_{c}^{-1}[u](x)=\int_{0}^{1} t^{c-1} u(t x) d t,
$$

where $r=|x|$ and $c \geq 0$ is a constant. Note that the operator $\Gamma_{0}^{-1}$ is not defined for functions $u(x)$ with $u(0) \neq 0$.

Note that in the class of harmonic functions in a ball the properties of operators $\Gamma_{c}^{1}$ and $\Gamma_{c}^{-1}$ with $c>0$ have previously been studied in the paper [8]. 
Lemma 1 Let $u(x)$ be a smooth function. Then for any $x \in \Omega$ one has

(1) if $c>0$, then

$$
\Gamma_{c}\left[\Gamma_{c}^{-1}[u]\right](x)=\Gamma_{c}^{-1}\left[\Gamma_{c}[u]\right](x)=u(x)
$$

(2) if $c=0$, then

$$
\Gamma_{0}^{-1}\left[\Gamma_{0}[u]\right](x)=u(x)-u(0)
$$

(3) if $c=0$ and $u(0)=0$, then

$$
\Gamma_{0}\left[\Gamma_{0}^{-1}[u]\right](x)=u(x)
$$

Proof Let $c \geq 0$. Then

$$
\int_{0}^{1} \frac{d}{d t}\left[t^{c} u(t x)\right] d t=\int_{0}^{1} t^{c-1}\left[c u(t x)+t \frac{d}{d t} u(t x)\right] d t=\int_{0}^{1} t^{c-1} \Gamma_{c}[u](t x) d t .
$$

Therefore, if $c>0$, then

$$
u(x)=\int_{0}^{1} t^{c-1} \Gamma_{c}[u](t x) d t=\Gamma_{c}^{-1}\left[\Gamma_{c}[u]\right](x)
$$

and if $c=0$, then

$$
u(x)-u(0)=\int_{0}^{1} t^{-1} \Gamma_{0}[u](t x) d t .
$$

Hence, equality (5) and the second equality of (4) are proved.

As we noted above, if $u(0)=0$, then the expression $\Gamma_{0}^{-1}[u](x)$ is defined. Now apply the operator $\Gamma_{0}$ to this expression. Then

$$
\Gamma_{0}\left[\Gamma_{0}^{-1}[u]\right](x)=\int_{0}^{1} t^{-1} \Gamma_{0}[u](t x) d t .
$$

But due to equality (7) and the condition $u(0)=0$, the last expression is equal to $u(x)$. Hence, equality (6) is proved. The first equality in (4) can be proved in the same way.

The following statement can be proved by a direct calculation.

Lemma 2 Let $u(x)$ be a smooth function. Then for any $x \in \Omega$ one has

(1) if $c \geq 0$, then

$$
\Delta \Gamma_{c}[u](x)=\Gamma_{c+2}[\Delta u](x)
$$

(2) if $c>0$, then

$$
\Delta \Gamma_{c}^{-1}[u](x)=\Gamma_{c+2}^{-1}[\Delta u](x)
$$


(3) if $c=0$ and $u(0)=0$, then

$$
\Delta \Gamma_{0}^{-1}[u](x)=\Gamma_{2}^{-1}[\Delta u](x)
$$

Corollary 1 Let $u(x)$ be a smooth function. Then for any $x \in \Omega$ one has

(1) if $c \geq 0$, then

$$
\Delta^{m} \Gamma_{c}[u](x)=\Gamma_{c+2 m}\left[\Delta^{m} u\right](x)
$$

(2) if $c>0$, or $c=0$ and $u(0)=0$, then

$$
\Delta^{m} \Gamma_{c}^{-1}[u](x)=\Gamma_{c+2 m}^{-1}\left[\Delta^{m} u\right](x) .
$$

\section{Some properties of the solutions of the Dirichlet problem}

Let $v(x)$ be a solution of the Dirichlet problem

$$
\begin{cases}(-\Delta)^{m} v(x)=g_{1}(x), & x \in \Omega, \\ \frac{\partial^{k-1} v}{\partial v^{k-1}}(x)=0, & k=1,2, \ldots, m, x \in \partial \Omega .\end{cases}
$$

It is known (see, for example, [5-7]) that if $g_{1}(x)$ is a sufficiently smooth function, then the solution of problem (10) exists, it is unique and has the form

$$
v(x)=\int_{\Omega} G_{m, n}(x, y) g_{1}(y) d y,
$$

where $G_{m, n}(x, y)$ is the Green function of Dirichlet problem (10).

We make use of the following explicit form of the Green function [5]

if $n$ is odd, or even and $n>2 m$, then

$$
\begin{aligned}
G_{m, n}(x, y)= & d_{m, n}\left[|x-y|^{2 m-n}-|x| y\left|-\frac{y}{|y|}\right|^{2 m-n}\right. \\
& -\sum_{k=1}^{m-1} \frac{(-1)^{k}}{k !}\left(m-\frac{n}{2}\right) \cdots\left(m-\frac{n}{2}-k+1\right) \\
& \left.\times|x| y\left|-\frac{y}{|y|}\right|^{2 m-n-2 k}\left(1-|x|^{2}\right)^{k}\left(1-|y|^{2}\right)^{k}\right],
\end{aligned}
$$

where

$$
d_{m, n}=\frac{(-1)^{m} \Gamma\left(\frac{n}{2}-m\right)}{\pi^{\frac{n}{2}} 4^{m}(m-1) !}
$$

if $n$ is even and $n \leq 2 m$, then

$$
\begin{aligned}
G_{m, n}(x, y)= & d_{m, n}\left[|x-y|^{2 m-n}\left(\ln |x-y|^{2}-\ln |x| y\left|-\frac{y}{|y|}\right|^{2}\right)\right. \\
& \left.+\sum_{k=1}^{m-1}|x| y\left|-\frac{y}{|y|}\right|^{2 m-n-2 k}\left(1-|x|^{2}\right)^{k}\left(1-|y|^{2}\right)^{k} \sum_{j=\max (k-n / 2,0)}^{\min (m-n / 2, k-1)} \frac{(-1)^{j}}{k-j} C_{m-n / 2}^{j}\right],
\end{aligned}
$$


where

$$
d_{m, n}=\frac{(-1)^{m-n / 2}}{2 \pi^{\frac{n}{2}} 4^{m}(m-n / 2) !(m-1) !} .
$$

Lemma 3 Let $g_{1}(x)=\Gamma_{2 m}[g](x)$ in Dirichlet problem (10), and let $v(x)$ be the unique solution of this problem. Then $v(0)=0$ if and only if

$$
\int_{\Omega}\left(1-|x|^{2}\right)^{m-1} g(x) d x=0 .
$$

Proof Let $v(x)$ be the solution of problem (10). Then it has the form (11). To use the explicit form of the Green function, we shall deal only with the case $n$ is odd or even and $n>2 m$, the other cases being exactly similar. So, if $v(0)=0$, then from (11) one has

$$
\int_{\Omega}\left[|y|^{2 m-n}-1-\sum_{k=1}^{m-1} \frac{(-1)^{k}}{k !}\left(m-\frac{n}{2}\right) \cdots\left(m-\frac{n}{2}-k+1\right)\left(1-|y|^{2}\right)^{k}\right] g_{1}(y) d y=0 .
$$

If we denote $\rho=|y|$ and $\xi=\frac{y}{|y|}$, then the last integral can be rewritten as

$$
\begin{aligned}
& \int_{|\xi|=1} \int_{0}^{1} \rho^{n-1}\left[\rho^{2 m-n}-1-\sum_{k=1}^{m-1} \frac{(-1)^{k}}{k !}\left(m-\frac{n}{2}\right) \cdots\left(m-\frac{n}{2}-k+1\right)\left(1-\rho^{2}\right)^{k}\right] \\
& \times g_{1}(\rho, \xi) d \rho d \xi=\int_{|\xi|=1} I(\xi) d \xi .
\end{aligned}
$$

Now we consider the inner integral $I(\xi)$. Noting that $g_{1}(\rho, \xi)=\left(\rho \frac{\partial}{\partial \rho}+2 m\right) g(\rho, \xi)$, we introduce the following two integrals:

$$
\begin{aligned}
I_{1}(\xi)= & \int_{0}^{1} \rho^{n-1}\left[2 m \rho^{2 m-n}-2 m-2 m \sum_{k=1}^{m-1} \frac{(-1)^{k}}{k !}\left(m-\frac{n}{2}\right) \cdots\left(m-\frac{n}{2}-k+1\right)\right. \\
& \left.\times\left(1-\rho^{2}\right)^{k}\right] g(\rho, \xi) d \rho, \\
I_{2}(\xi)= & \int_{0}^{1} \rho^{n}\left[\rho^{2 m-n}-1-\sum_{k=1}^{m-1} \frac{(-1)^{k}}{k !}\left(m-\frac{n}{2}\right) \cdots\left(m-\frac{n}{2}-k+1\right)\left(1-\rho^{2}\right)^{k}\right] \\
& \times \frac{\partial}{\partial \rho} g(\rho, \xi) d \rho .
\end{aligned}
$$

Obviously, $I(\xi)=I_{1}(\xi)+I_{2}(\xi)$.

Integrating by part in the integral $I_{2}(\xi)$, we obtain

$$
\begin{aligned}
I_{2}(\xi)= & \int_{0}^{1} \rho^{n-1}\left[-n \rho^{2 m-n}+n\right. \\
& +n \cdot\left[\sum_{k=1}^{m-1} \frac{(-1)^{k}}{k !}\left(m-\frac{n}{2}\right) \cdots\left(m-\frac{n}{2}-k+1\right)\left(1-\rho^{2}\right)^{k}\right]-(2 m-n) \rho^{2 m-n} \\
& \left.-2 \rho^{2}\left[\sum_{k=1}^{m-1} \frac{(-1)^{k}}{k !} k\left(m-\frac{n}{2}\right) \cdots\left(m-\frac{n}{2}-k+1\right)\left(1-\rho^{2}\right)^{k-1}\right]\right] g(\rho, \xi) d \rho .
\end{aligned}
$$


Therefore

$$
I_{1}(\xi)+I_{2}(\xi)=\int_{0}^{1} \rho^{n-1} S_{m-1}(\rho) g(\rho, \xi) d \rho,
$$

where

$$
\begin{aligned}
S_{j}(\rho)= & n-2 m-2 \rho^{2} \sum_{k=1}^{j} \frac{(-1)^{k}}{(k-1) !}\left(m-\frac{n}{2}\right) \cdots\left(m-\frac{n}{2}-k+1\right)\left(1-\rho^{2}\right)^{k-1} \\
& +(n-2 m) \sum_{k=1}^{j} \frac{(-1)^{k}}{k !}\left(m-\frac{n}{2}\right) \cdots\left(m-\frac{n}{2}-k+1\right)\left(1-\rho^{2}\right)^{k}, \quad 1 \leq j \leq m-1 .
\end{aligned}
$$

It is not hard to prove by induction that

$$
S_{j}(\rho)=\frac{(n-2 m) \cdots(n-2(m-j))}{2^{j}} \cdot \frac{\left(1-\rho^{2}\right)^{j}}{j !} .
$$

Indeed, if $j=1$, then

$$
\begin{aligned}
S_{1}(\rho) & =n-2 m-2 \rho^{2}(-1)\left(m-\frac{n}{2}\right)+(n-2 m)(-1)\left(m-\frac{n}{2}\right)\left(1-\rho^{2}\right) \\
& =(n-2 m)\left(1-\rho^{2}\right)+(n-2 m) \cdot \frac{n-2 m}{2}\left(1-\rho^{2}\right) \\
& =\frac{(n-2 m)(n-2(m-1))}{2}\left(1-\rho^{2}\right) .
\end{aligned}
$$

Now let us suppose that (13) holds true for some $j$ and prove it for $j+1$. We have

$$
\begin{aligned}
S_{j+1}(\rho)= & \frac{(n-2 m) \cdots(n-2(m-j))}{2^{j}} \cdot \frac{\left(1-\rho^{2}\right)^{j}}{j !} \\
& -\frac{2}{j !} \cdot \frac{n-2 m}{2} \cdots \frac{n-2(m-j)}{2}\left(1-\rho^{2}\right)^{j} \rho^{2} \\
& +\frac{n-2 m}{(j+1) !} \cdot \frac{n-2 m}{2} \cdots \frac{n-2(m-j)}{2}\left(1-\rho^{2}\right)^{j+1} \\
= & \frac{(n-2 m) \cdots(n-2(m-j))}{2^{j}} \cdot \frac{\left(1-\rho^{2}\right)^{j+1}}{j !} \\
& +\frac{n-2 m}{2(j+1)} \cdot \frac{(n-2 m) \cdots(n-2(m-j))}{2^{j}} \cdot \frac{\left(1-\rho^{2}\right)^{j+1}}{j !} \\
= & \frac{(n-2 m) \cdots(n-2(m-j))}{2^{j}} \cdot \frac{n-2(m-j-1)}{2} \cdot \frac{\left(1-\rho^{2}\right)^{j+1}}{(j+1) !} .
\end{aligned}
$$

Thus equality (13) holds true for any $j=1,2, \ldots, m-1$. In particular,

$$
S_{m-1}(\rho)=\frac{(n-2 m) \cdots(n-2)}{2^{m-1}} \cdot \frac{\left(1-\rho^{2}\right)^{m-1}}{(m-1) !}=a_{n, m}\left(1-\rho^{2}\right)^{m-1},
$$

where

$$
a_{n, m}=\frac{(n-2 m) \cdots(n-2)}{2^{m-1} \cdot(m-1) !} .
$$


Therefore

$$
0=\int_{|\xi|=1} I(\xi) d \xi=a_{n, m} \int_{|\xi|=1} \int_{0}^{1} \rho^{n-1}\left(1-\rho^{2}\right)^{m-1} g(\rho, \xi) d \rho d \xi,
$$

and going back to the Cartesian coordinate system, we have

$$
\int_{\Omega}\left(1-|x|^{2}\right)^{m-1} g(x) d x=0 .
$$

\section{The Neumann problem with homogeneous boundary conditions}

In this section we study problem (1), (2) with homogeneous boundary conditions.

Theorem 1 Let $g(x)$ be sufficiently smooth. Then the necessary and sufficient solvability condition for Neumann problem (1), (2) has the form (12).

If a solution exists, then it is unique up to a constant and can be represented as

$$
u(x)=C+\Gamma_{0}^{-1}[v](x),
$$

where $v(x)$ is the solution of Dirichlet problem (10) with the right-hand side $g_{1}(x)=$ $\Gamma_{2 m}[g](x)$, which satisfies the additional condition $v(0)=0$.

Proof Let a solution of problem (1), (2) exist and let $u(x)$ be this solution. We apply an operator $\Gamma_{0}$ to a function $u(x)$ and denote $v(x)=\Gamma_{0}[u](x)$. Now we obtain the conditions for the function $v(x)$.

Obviously, $v(0)=0$. If we apply the operator $(-\Delta)^{m}$ to $v(x)$, then by virtue of $(8)$ we have

$$
(-\Delta)^{m} v(x)=\Gamma_{2 m}\left[(-\Delta)^{m} u\right](x)=\Gamma_{2 m}[g](x)=g_{1}(x) .
$$

Further, since

$$
r \frac{\partial u}{\partial r}(x)=\frac{\partial u}{\partial v}(x), \quad x \in \partial \Omega,
$$

then

$$
v(x)=\frac{\partial u}{\partial v}(x)=0, \quad x \in \partial \Omega
$$

It is not hard to verify that for any $k=1,2, \ldots$ and all $x \in \partial \Omega$ one has [9]

$$
\frac{\partial^{k} v}{\partial v^{k}}(x)=r \frac{\partial}{\partial r}\left(r \frac{\partial}{\partial r}-1\right) \cdots\left(r \frac{\partial}{\partial r}-k+1\right) v(x)=r^{k} \frac{\partial^{k} v}{\partial r^{k}}(x) .
$$

Therefore from homogeneous conditions (2) we finally have

$$
\frac{\partial^{k} v}{\partial v^{k}}(x)=0, \quad k=1,2, \ldots, m-1, x \in \partial \Omega
$$


Thus, if $u(x)$ is a solution of problem (1), (2) with homogeneous boundary conditions, then the function $v(x)=\Gamma_{0}[u](x)$ is the solution of Dirichlet problem (10) with the righthand side

$$
g_{1}(x)=\left(r \frac{\partial}{\partial r}+2 m\right) g(x)=\Gamma_{2 m}[g](x) .
$$

Moreover, the function $v(x)$ satisfies the condition $v(0)=0$ and according to Lemma 3, the necessary condition for this is (12). Hence, if a solution of problem (1), (2) exists, then it is necessary for condition (12) to be satisfied.

Now we prove that if condition (12) is satisfied, then the solution of problem (1), (2) with homogeneous boundary conditions exists.

Indeed, if (12) is satisfied, then according to Lemma 3 the solution $v(x)$ of Dirichlet problem (10) with $g_{1}(x)=\Gamma_{2 m}[g](x)$ exists and $v(0)=0$.

Therefore, we may apply the operator $\Gamma_{0}^{-1}$ to $v(x)$ and consider the function $u(x)=C+$ $\Gamma_{0}^{-1}[v](x)$. It is not hard to show that this function is the solution of problem (1), (2).

Indeed, by virtue of (9) one has

$$
(-\Delta)^{m} u(x)=\Gamma_{2 m}^{-1}\left[(-\Delta)^{m} v\right](x)=\Gamma_{2 m}^{-1}\left[\Gamma_{2 m}[g]\right](x)=g(x), \quad x \in \Omega .
$$

Since $\Gamma_{0}[u](x)=\Gamma_{0}\left[\Gamma_{0}^{-1}[v]\right](x)=v(x)$, then one can show as above that the function $u(x)$ satisfies all homogeneous boundary conditions.

\section{The Neumann problem for the homogeneous equation}

In the present section we consider Neumann problem (1), (2) with $g(x) \equiv 0$.

Let $A$ be the following matrix

$$
\left(\begin{array}{ccccc}
1 & 1 & 1 & \cdots & 1 \\
0 & 2^{[1]} & 4^{[1]} & \cdots & (2 m-2)^{[1]} \\
0 & 2^{[2]} & 4^{[2]} & \cdots & (2 m-2)^{[2]} \\
\cdots & \cdots & \cdots & \cdots & \cdots \\
0 & 2^{[m-1]} & 4^{[m-1]} & \cdots & (2 m-2)^{[m-1]}
\end{array}\right),
$$

where $j^{[k]}=j(j-1) \cdots(j-k+1), j^{[1]}=j$. Note that $j^{[k]}=0$ if $k>j$. Denote by $\Delta_{j}, j=1,2, \ldots, m$, the determinant of the matrix obtained from $A$ by deleting the elements of the first column and the $j$ th row. Obviously, $|A|=\operatorname{det} A=\Delta_{1}$.

Let $w(x)$ be a solution of the following Dirichlet problem with sufficiently smooth boundary functions $f_{k}(x)$ :

$$
\begin{cases}(-\Delta)^{m} w(x)=0, & x \in \Omega, \\ \frac{\partial^{k-1} w}{\partial v^{k-1}}(x)=f_{k}(x), & k=1,2, \ldots, m, x \in \partial \Omega .\end{cases}
$$

Theorem 2 Let $g(x) \equiv 0$ and $\varphi_{k}(x), k=1,2, \ldots, m$, be sufficiently smooth functions. Then the necessary and sufficient solvability condition for Neumann problem (1), (2) has the form

$$
\sum_{k=1}^{m} \int_{\partial \Omega}(-1)^{k+1} \Delta_{k}\left[\varphi_{k}(x)+(k-1) \varphi_{k-1}(x)\right] d S_{x}=0,
$$

where $\varphi_{0}(x)=0$. 
If a solution exists, then it is unique up to a constant and can be represented as

$$
u(x)=C+\Gamma_{0}^{-1}[w](x)
$$

where $w(x)$ is the solution of Dirichlet problem (15) with boundary functions $f_{1}(x)=$ $\varphi_{1}(x), f_{k}(x)=\varphi_{k}(x)+(k-1) \varphi_{k-1}(x), k=2,3, \ldots, m$, which satisfies the additional condition $w(0)=0$.

Proof Let a solution of problem (1), (2) exist and let $u(x)$ be this solution. We apply an operator $\Gamma_{0}$ to a function $u(x)$ and denote $w(x)=\Gamma_{0}[u](x)$. Now we prove that the function $w(x)$ is the solution of Dirichlet problem (15) with the additional condition $w(0)=0$.

From the properties of the operator $\Gamma_{0}$ we have $(-\Delta)^{m} w(x)=0, x \in \Omega$. By virtue of the following formula [9]

$$
\frac{\partial^{j} u}{\partial \nu^{j}}=\left(r \frac{\partial}{\partial r}-j+1\right) \cdots\left(r \frac{\partial}{\partial r}-1\right) r \frac{\partial u}{\partial r},
$$

one has for $x \in \partial \Omega$

$$
\begin{aligned}
& w(x)=\varphi_{1}(x), \\
& \left(r \frac{\partial}{\partial r}-j+1\right) \cdots\left(r \frac{\partial}{\partial r}-1\right) w(x)=\varphi_{j}(x), \quad j=2,3, \ldots, m .
\end{aligned}
$$

We rewrite these conditions in a more convenient form. To do this we first consider the last two of them:

$$
\begin{aligned}
& \left(r \frac{\partial}{\partial r}-m+2\right) \cdots\left(r \frac{\partial}{\partial r}-1\right)\left(r \frac{\partial}{\partial r}-(m-1)\right) w(x)=\varphi_{m}(x), \\
& \left(r \frac{\partial}{\partial r}-m+2\right) \cdots\left(r \frac{\partial}{\partial r}-1\right) w(x)=\varphi_{m-1}(x) .
\end{aligned}
$$

We multiply expression (19) by $m-1$ and sum to (18). Then, making use of (17), we obtain

$$
\begin{aligned}
& \left(r \frac{\partial}{\partial r}-m+2\right) \cdots\left(r \frac{\partial}{\partial r}-1\right) r \frac{\partial w(x)}{\partial r} \\
& \quad=\frac{\partial^{m-1} w(x)}{\partial \nu^{m-1}}=\varphi_{m}(x)+(m-1) \varphi_{m-1}(x)=f_{m}(x), \quad x \in \partial \Omega .
\end{aligned}
$$

Further, by repeating this argument for all $1 \leq j \leq m-1$, we get

$$
\frac{\partial^{j} w(x)}{\partial v^{j}}=\varphi_{j+1}(x)+j \varphi_{j}(x)=f_{j+1}(x), \quad x \in \partial \Omega .
$$

Thus, if $u(x)$ is the solution of Neumann problem (1), (2), then the function $w(x)=$ $\Gamma_{0}[u](x)$ will be the solution of Dirichlet problem (15) with the additional condition $w(0)=0$. Note that, under the conditions of Theorem 2, the solution of problem (15) exists and it is unique (see, for example, [10]). 
Next we find the conditions to the boundary functions $\varphi_{k}(x)$, which guarantee the equality $w(0)=0$. Making use of the Almansi formula (see, for example, [11], p.188) we write the solution of problem (15) as

$$
w(x)=w_{0}(x)+r^{2} w_{1}(x)+r^{4} w_{2}(x)+\cdots+r^{2 m-2} w_{m-1}(x),
$$

where $w_{j}(x)$ are harmonic functions in the ball $\Omega$. Obviously, $w(0)=0$ if and only if $w_{0}(0)=0$.

Substituting function (20) into the boundary condition of (15) and integrating over the sphere, taking into account the equalities

$$
\int_{\partial \Omega} \frac{\partial^{k} w_{j}(x)}{\partial v^{k}} d S_{x}=\left\{\begin{array}{ll}
\omega_{n} w_{j}(0), & k=0, \\
0, & k \neq 0,
\end{array} \quad \omega_{n}\right. \text { is the area of the unit sphere }
$$

we get the system of equations

$$
\begin{aligned}
& w_{0}(0)+w_{1}(0)+w_{2}(0)+\cdots+w_{m-1}(0)=b_{1}, \\
& 0 \cdot w_{0}(0)+2 w_{1}(0)+4 w_{2}(0)+\cdots+(2 m-2) w_{m-1}(0)=b_{2}, \\
& 0 \cdot w_{0}(0)+2 w_{1}(0)+4 \cdot 3 w_{2}(0)+\cdots+(2 m-2)(2 m-3) w_{m-1}(0)=b_{3}, \\
& \cdots \\
& 0 \cdot w_{0}(0)+2^{[m-1]} w_{1}(0)+4^{[m-1]} w_{2}(0)+\cdots+(2 m-2)^{[m-1]} w_{m-1}(0)=b_{m},
\end{aligned}
$$

where

$$
b_{k}=\frac{1}{\omega_{n}} \int_{\partial \Omega}\left[\varphi_{k}(x)+(k-1) \varphi_{k-1}(x)\right] d S_{x}, \quad k=1,2, \ldots, m,
$$

and $\varphi_{0}(x) \equiv 0$. The matrix of this system is matrix $A$, defined by (14). As we noted above, $|A|=\operatorname{det} A=\Delta_{1}$. By reducing to the Vandermonde determinant, it is not hard to find the value of this determinant; one has $\Delta_{1}=2^{m-1}(m-1) !(2 m-4) ! ! \cdots 4 ! ! 2 ! !$.

Making use of Cramer's rule, we find $w_{0}(0)$ from the above system of equations: $w_{0}(0)=$ $\frac{|B|}{|A|}$, where $|B|$ is the determinant of the following matrix

$$
\left(\begin{array}{ccccc}
b_{1} & 1 & 1 & \cdots & 1 \\
b_{2} & 2^{[1]} & 4^{[1]} & \cdots & (2 m-2)^{[1]} \\
b_{3} & 2^{[2]} & 4^{[2]} & \cdots & (2 m-2)^{[2]} \\
\cdots & \cdots & \cdots & \cdots & \cdots \\
b_{m} & 2^{[m-1]} & 4^{[m-1]} & \cdots & (2 m-2)^{[m-1]}
\end{array}\right) .
$$

Obviously,

$$
|B|=\sum_{k=1}^{m}(-1)^{k+1} b_{k} \Delta_{k}
$$

where determinants $\Delta_{k}, k=1,2, \ldots, m$, are defined above. Therefore, the equality $w_{0}(0)=0$ holds if and only if $\sum_{k=1}^{m}(-1)^{k+1} b_{k} \Delta_{k}=0$. But by the definition of $b_{k}$, this condition is equivalent to (16). 
Thus, if the solution of the considered Neumann problem exists, then necessarily condition (16) holds.

We now prove the converse, i.e., if (16) holds, then the solution of the Neumann problem exists.

Let $w(x)$ be the solution of Dirichlet problem (15). If condition (16) holds, then $w(0)=0$ and we may consider the function

$$
u(x)=C+\Gamma_{0}^{-1}[w](x)=C+\int_{0}^{1} s^{-1} w(s r \theta) d s, \quad x=r \theta,
$$

and prove that this function is in fact the solution of the Neumann problem.

Indeed, after changing of variable $s r=\xi$, the last integral can be written as

$$
u(x)=C+\int_{0}^{r} \frac{w(\xi \theta)}{\xi} d \xi
$$

Therefore,

$$
r \frac{\partial u(x)}{\partial r}=r \frac{\partial}{\partial r}\left(\int_{0}^{r} \frac{w(\xi \theta)}{\xi} d \xi\right)=w(x) .
$$

In the subsequent discussions, we use formulas (17) and (21) and assume that $x \in \partial \Omega$. So, we have

$$
\frac{\partial u(x)}{\partial v}=r \frac{\partial u(x)}{\partial r}=w(x)=f_{1}(x)=\varphi_{1}(x) .
$$

Further, for the second derivative one has

$$
\frac{\partial^{2} u(x)}{\partial v^{2}}=\left(r \frac{\partial}{\partial r}-1\right) r \frac{\partial u(x)}{\partial r}=\left(r \frac{\partial}{\partial r}-1\right) w(x) .
$$

Then

$$
\begin{aligned}
\frac{\partial^{2} u(x)}{\partial v^{2}}+\frac{\partial u(x)}{\partial v} & =\left(r \frac{\partial}{\partial r}-1\right) r \frac{\partial u(x)}{\partial r}+r \frac{\partial u(x)}{\partial r}=r \frac{\partial w(x)}{\partial r} \\
& =\frac{\partial w(x)}{\partial v}=f_{2}(x)=\varphi_{2}(x)+\varphi_{1}(x) .
\end{aligned}
$$

Hence

$$
\frac{\partial^{2} u(x)}{\partial v^{2}}=\varphi_{2}(x)+\varphi_{1}(x)-\frac{\partial u(x)}{\partial v}=\varphi_{2}(x) .
$$

Using the same argument, we have for any $j=2,3, \ldots, m$

$$
\begin{aligned}
\frac{\partial^{j} u(x)}{\partial v^{j}} & =\left(r \frac{\partial}{\partial r}-j+1\right) \cdots\left(r \frac{\partial}{\partial r}-1\right) r \frac{\partial u(x)}{\partial r} \\
& =\left(r \frac{\partial}{\partial r}-j+1\right) \cdots\left(r \frac{\partial}{\partial r}-1\right) w(x) .
\end{aligned}
$$


Consequently,

$$
\begin{aligned}
\frac{\partial^{j} u(x)}{\partial \nu^{j}}+(j-1) \frac{\partial^{j-1} u(x)}{\partial \nu^{j-1}}= & \left(r \frac{\partial}{\partial r}-(j-1)\right)\left(r \frac{\partial}{\partial r}-(j-2)\right) \cdots\left(r \frac{\partial}{\partial r}-1\right) w(x) \\
& +(j-1)\left(r \frac{\partial}{\partial r}-(j-2)\right) \cdots\left(r \frac{\partial}{\partial r}-1\right) w(x) \\
= & \frac{\partial^{j-1} w(x)}{\partial \nu^{j-1}}=f_{j}(x)=\varphi_{j}(x)+(j-1) \varphi_{j-1}(x) .
\end{aligned}
$$

Therefore, finally we have by induction

$$
\begin{aligned}
\frac{\partial^{j} u(x)}{\partial v^{j}} & =\varphi_{j}(x)+(j-1) \varphi_{j-1}(x)-(j-1) \frac{\partial^{j-1} u(x)}{\partial \nu^{j-1}} \\
& =\varphi_{j}(x)+(j-1) \varphi_{j-1}(x)-(j-1) \varphi_{j-1}(x)=\varphi_{j}(x) .
\end{aligned}
$$

\section{The Neumann problem in the general case}

In this final section we consider Neumann problem (1), (2) in the case when both the equation and the conditions are non-homogeneous.

Let the solution of problem (1), (2) exist and denote this solution by $u(x)$. Apply the operator $\Gamma_{0}$ to $u(x)$ and put $z(x)=\Gamma_{0}[u](x)$. Then the function $z(x)$ is a solution of the following Dirichlet problem:

$$
\begin{cases}(-\Delta)^{m} z(x)=g_{1}(x), & x \in \Omega, \\ \frac{\partial^{k-1} z}{\partial \nu^{k-1}}(x)=\varphi_{k}(x)+(k-1) \varphi_{k-1}(x), & k=1,2, \ldots, m, x \in \partial \Omega,\end{cases}
$$

where $g_{1}(x)=\Gamma_{2 m}[g](x), \varphi_{0}(x) \equiv 0$. Moreover, by definition $z(x)$ satisfies the additional condition $z(0)=0$. Since functions $g_{1}$ and $\varphi_{k}$ are sufficiently smooth, then the solution of problem (22) exists and it is unique.

Next we find the conditions to functions $g(x)$ and $\varphi_{k}(x)$, which guarantee the equality $z(0)=0$. To do this we present $z(x)$ as

$$
z(x)=v(x)+w(x)
$$

where $v(x)$ and $w(x)$ are the considered above solutions of the corresponding Dirichlet problems (10) and (15). Obviously, $z(0)=0$ if and only if $v(0)+w(0)=0$.

We represent the functions $v(x)$ and $w(x)$, according to (11) and (20), in the form

$$
\begin{aligned}
& v(x)=\int_{\Omega} G_{m, n}(x, y) g_{1}(y) d y, \\
& w(x)=w_{0}(x)+r^{2} w_{1}(x)+r^{4} w_{2}(x)+\cdots+r^{2 m-2} w_{m-1}(x) .
\end{aligned}
$$

Then

$$
\begin{aligned}
& v(0)=\int_{\Omega} G_{m, n}(0, y) g_{1}(y) d y=C_{n, m} \int_{\Omega}\left(1-|y|^{2}\right)^{m-1} g(y) d y, \\
& w(0)=w_{0}(0)=-\frac{1}{\omega_{n}|A|} \sum_{k=1}^{m} \int_{\partial \Omega}(-1)^{k} \Delta_{k}\left[\varphi_{k}(x)+(k-1) \varphi_{k-1}(x)\right] d S_{x},
\end{aligned}
$$


where

$$
C_{n, m}=d_{n, m} a_{n, m}=\frac{(-1)^{m} \Gamma\left(\frac{n}{2}-m\right)}{\pi^{\frac{n}{2}} 4^{m}(m-1) !} \cdot \frac{(n-2 m) \cdots(n-2)}{2^{m-1}(m-1) !} .
$$

It is not hard to see that using the formula $\omega_{n}=\frac{2 \pi^{n / 2}}{\Gamma(n / 2)}$ we can simplify $C_{n, m}$ and obtain

$$
C_{n, m}=\frac{1}{\omega_{n}} \cdot \frac{(-1)^{m}}{\left(2^{m-1}(m-1) !\right)^{2}} .
$$

Since $|A|=\Delta_{1}=2^{m-1}(m-1) !(2 m-4) ! ! \cdots 4 ! ! 2 ! !$, the condition $v(0)+w(0)=0$ has the form

$$
\begin{aligned}
& \int_{\Omega}\left(1-|x|^{2}\right)^{m-1} g(x) d x \\
& \quad=\frac{2^{m-1}(m-1) !}{(2 m-4) ! ! \cdots 4 ! ! 2 ! !} \sum_{k=1}^{m} \int_{\partial \Omega}(-1)^{k} \Delta_{k}\left[\varphi_{k}(x)+(k-1) \varphi_{k-1}(x)\right] d S_{x},
\end{aligned}
$$

where $\varphi_{0}(x)=0$.

Thus, we proved the following statement on the necessary and sufficient solvability condition for the general Neumann boundary value problem.

Theorem 3 Let $\varphi_{k}(x), k=1,2, \ldots, m$ and $g(x)$ be sufficiently smooth. Then the necessary and sufficient solvability condition for Neumann boundary value problem (1), (2) has the form (23).

If a solution exists, then it is unique up to a constant and can be represented as

$$
u(x)=C+\Gamma_{0}^{-1}[z](x),
$$

where $z(x)$ is the solution of Dirichlet problem (22) with the right-hand side $g_{1}(x)=$ $\Gamma_{2 m}[g](x)$, which satisfies the additional condition $z(0)=0$.

Example 1 Let us consider the biharmonic equation, i.e., $m=2$.

In this case

$$
A=\left(\begin{array}{ll}
1 & 1 \\
0 & 2
\end{array}\right), \quad \Delta_{1}=2, \quad \Delta_{2}=1 .
$$

Then

$$
\sum_{k=1}^{2} \int_{\partial \Omega}(-1)^{k} \Delta_{k}\left[\varphi_{k}(x)+(k-1) \varphi_{k-1}(x)\right] d S_{x}=\int_{\partial \Omega}\left[\varphi_{1}(x)-\varphi_{2}(x)\right] d S_{x} .
$$

Therefore the solvability condition has the form

$$
\int_{\Omega}\left(1-|x|^{2}\right) g(x) d x=2 \int_{\partial \Omega}\left[\varphi_{2}(x)-\varphi_{1}(x)\right] d S_{x}
$$

i.e., this condition coincides with the result of the paper [3]. 
Example 2 Let us consider the so-called three-harmonic equation, i.e., $m=3$.

In this case

$$
A=\left(\begin{array}{ccc}
1 & 1 & 1 \\
0 & 2 & 4 \\
0 & 2 & 12
\end{array}\right), \quad \Delta_{1}=16, \quad \Delta_{2}=10, \quad \Delta_{3}=2
$$

Then

$$
\begin{aligned}
& \sum_{k=1}^{3} \int_{\partial \Omega}(-1)^{k} \Delta_{k}\left[\varphi_{k}(x)+(k-1) \varphi_{k-1}(x)\right] d S_{x} \\
& \quad=2 \int_{\partial \Omega}\left[3 \varphi_{1}(x)-3 \varphi_{2}(x)+\varphi_{3}(x)\right] d S_{x} .
\end{aligned}
$$

Therefore the solvability condition has the form

$$
\int_{\Omega}\left(1-|x|^{2}\right)^{2} g(x) d x=8 \int_{\partial \Omega}\left[3 \varphi_{1}(x)-3 \varphi_{2}(x)+\varphi_{3}(x)\right] d S_{x} .
$$

Remark 1 Let $0<\lambda<1$. Obviously, if $g(x) \in C^{\lambda+1}(\Omega)$, then $g_{1}(x)=\Gamma_{2 m}[g](x) \in C^{\lambda}(\Omega)$. Therefore, if we suppose that $g(x) \in C^{\lambda+1}(\Omega)$ and $\varphi_{k}(x) \in C^{\lambda+m+1-k}(\partial \Omega), k=1,2, \ldots, m$, then all the considered boundary value problems have solutions and these solutions are unique (see, for example, [2]).

\section{Competing interests}

The authors declare that they have no competing interests.

\section{Authors' contributions}

Both authors completed the paper together. Both authors read and approved the final manuscript.

\section{Author details}

${ }^{1}$ Department of Mathematics, Akhmet Yasawi International Kazakh-Turkish University, B. Sattarkhanov street, 29, Turkistan, 161200, Kazakhstan. ${ }^{2}$ Institute of Mathematics National University of Uzbekistan, Hodjaeva st., 29, Tashkent, 100125, Uzbekistan.

\section{Acknowledgements}

This work has been supported by the MON Republic of Kazakhstan under Research Grant 0830/GF2 and the Ministry of Higher and Secondary Special Education of Uzbekistan under Research Grant F4-FA-F010.

Received: 23 April 2013 Accepted: 19 June 2013 Published: 5 July 2013

\section{References}

1. Vladimirov, VC: Equations of Mathematical Physics. Nauka, Moscow (1974) (in Russian)

2. Kanguzhin, BE, Koshanov, BD: Necessary and sufficient conditions of solvability of boundary value problems for inhomogeneous polyharmonic equation in a ball. Ufa Math. J. 2(2), 41-52 (2010) (in Russian)

3. Karachik, W, Turmetov, BK, Bekayeva, AE: Solvability conditions of the Neumann boundary value problem for the biharmonic equation in the unit ball. Int. J. Pure Appl. Math. 81(3), 487-495 (2012)

4. Torebek, BT, Turmetov, BK: On solvability of a boundary value problem for the Poisson equation with the boundary operator of a fractional order. Bound. Value Probl. (2013). doi:10.1186/1687-2770-2013-93

5. Kal'menov, TS, Suragan, D: On a new method for constructing the green function of the Dirichlet problem for the polyharmonic equation. Differ. Equ. 48(3), 441-445 (2012)

6. Kal'menov, TS, Koshanov, BD, Nemchenko, MY: The Green function representation in the Dirichlet problem for polyharmonic equations in a ball. Dokl. Akad. Nauk 421(3), 305-307 (2008)

7. Kanguzhin, BE, Koshanov, BD: The Green function representation and properties in the Dirichlet problem for polyharmonic equations. Math. J. 8(1), 50-58 (2008)

8. Bavrin, II: Operators for harmonic functions and their applications. Differ. Uravn. 21(1), 9-15 (1985) (in Russian); English transl.: Differ. Equ. 21(1), 6-10 (1985) 
9. Karachik, W: Construction of polynomial solutions to some boundary value problems for Poisson's equation. Comput. Math. Math. Phys. 51(9), 1567-1587 (2011)

10. Agmon, S, Douglas, A, Nirenberg, L: Estimates near the boundary for solutions of elliptic partial differential equations satisfying general boundary conditions II. Commun. Pure Appl. Math. 17, 35-92 (1964)

11. Bitsadze, AV: Boundary Value Problems for Second Order Elliptic Equations. North-Holland, Amsterdam (1968)

doi:10.1186/1687-2770-2013-162

Cite this article as: Turmetov and Ashurov: On solvability of the Neumann boundary value problem for a non-homogeneous polyharmonic equation in a ball. Boundary Value Problems 2013 2013:162.

Submit your manuscript to a SpringerOpen ${ }^{\circ}$ journal and benefit from:

- Convenient online submission

- Rigorous peer review

- Immediate publication on acceptance

- Open access: articles freely available online

- High visibility within the field

- Retaining the copyright to your article

Submit your next manuscript at $>$ springeropen.com 